\title{
Molecular Identification and Prevalence of Sarcocystis Suihominis in Pork Meat Intended for Public Consumption
}

\author{
Alina FĂT*, Alexandra TĂBĂRAN, Sorin Daniel DAN, Oana REGET, Ionuţ CORDIȘ, Darius CORDEA, \\ Marian MIHAIU \\ ${ }^{1}$ Department of Animal Production and Food Safety, University of Agricultural Sciences and Veterinary \\ Medicine, Faculty of Veterinary Medicine, 3-5 Mănăştur Street, 400372, ClujNapoca, Romania \\ *corresponding author: fat.alinaioana@yahoo.com
}

Bulletin UASVM Veterinary Medicine 74(1) / 2017,

Print ISSN 1843-5270; Electronic ISSN 1843-5378

DOI:10.15835/buasvmcn-vm: 12536

\begin{abstract}
Protozoa of the genus Sarcocystisare among the most prevalent parasitic forms found in pork. In order to rapidly assess the risk that these protozoa produce in the human population, a molecular method to detect and identify Sarcocystissuihominis needs to be implemented. The study aimed at characterizing the prevalence of Sarcocystissuihominisin pork obtained in the traditional households through PCR - RFLP method. The material was represented by seventy nine samples of diaphragmatic pillars collected between the period October 2014 - December 2014 from two local sanitary veterinary units from Alba and Cluj. The samples were processed first by trichinelloscopic compression method. All the positive fragments for Sarcocystis spp. were examined through PCR-RFLP method. The method used in the present study utilized the AluI restriction enzyme and had successfully differentiated the Sarcocystissuihominis from other Sarcocystis spp. prevalent in pork meat. The inspection of compressed muscle tissue has detected the presence of Sarcocystissuihominis in a high percentage of investigated samples. The prevalence of Sarcocystissuihominis in the examined samples was $26.58 \%$. According to this result we can affirm that the risk of contamination in humans with Sarcocystissuihominis is high through consumption of contaminated pork meat, raised in the traditional system. In the present scientific research, we demonstrated that this method used for molecular identification of Sarcocystissuihominis has high accuracy and can be successfully applied for obtaining a certain diagnostic.
\end{abstract}

Keywords: meat, PCR, risk, Sarcocystis, traditional

\section{INTRODUCTION}

Sarcocystosis is one of the most prevalent parasitic diseases among wild and domestic animals in the world (Prakas and Butkausas, 2012). Sarcocystosis is widespread globally and evolve in all animal species, being conditioned by the action of multiple factors: geo-climatic, socioeconomic, growing conditions, diet, medication, species and age (Constantin, 2014).

Sarcocystis parasites belong to the phylum Apicomplexa, class Sporozoasida, order Eimerio- rina, family Sarcocystidae and genus Sarcocystis (Dubey and Odening, 2001).

Sarcocystis species are intracellular protozoan parasites with a requisite two-host life cycle based on a prey-predator (intermediate-definitive) host relationship (Fayer, 2004).

Muscular cysts of various Sarcocystis spp. are found in a broad spectrum of intermediate hosts, such as mammals (74\%), birds (14\%) and reptiles $(10 \%)$. Only $0.5 \%$ of the intermediate hosts are fish (Prakas and Butkausas, 2012). 
Sarcocystosis is a zoonotic and parasitic disease commonly seen in domestic animals such as pigs. Among these, Sarcocystis suihominis is important in terms of public health, as meat and meat products are the main source of infection in human beings. Therefore, the infection in human is produced through the ingestion of well-developed tissue cysts containing bradyzoites (Juyal and Bhatia, 1989). The early signs and symptoms of the infection with S. suihomonis acquired through the consumption of raw or undercooked infected pork meat include nausea, diarrhea and vomiting (Tenter, 1995).

The WHO reported (1981) that, about $50 \%$ of parasitic cysts found in muscles of cattle and pigs belong to the species $S$. hominis and $S$. suihominis, respectively (Acha and Szyfres, 2003). High prevalence of muscles sarcocystiosis in pigs was confirmed by data from India, where investigators found that S.suihominis and $S$. meischeriana have been determined in $47 \%$ and $43 \%$ of pigs, respectively (Saleque and Bhatia, 1991).

In pigs, the muscular cysts are found most commonly in cardiac, diaphragmatic and esophageal muscles. Cysts are white and cylindrical, ranging in size from a few micrometers to a few centimeters and may or may not be visible to the naked eye, being similar with a grain of rice (Cătoi, 2003).

A variety of molecular methods have been developed and used to detect and identify Sarcocystis spp. in order to assess the genetic diversity among this protozoan from different hosts. Ribosomal DNA sequences are the most common molecular markers used in Sarcocystis spp. differentiation (Dahlgren and Gjerde, 2008; Rosenthal et al., 2008; Xiang et al., 2009).

Molecular techniques such as PCR and its variants are widely used to genetically determine diversity among many organisms and species and have been applied to many phylogenetic and taxonomic works (Güçlü et al., 2004).

Another important technique used in the diagnosis of Sarcocystis spp. is the RFLP technique (restriction fragment length polymorphism analysis). This method is based on the fragmentation of the nucleic acids obtained from the PCR reaction. In the RFLP analysis, nucleic acids are digested by the restriction enzyme. After digestion, it realizes a specific pattern of fragments of nucleic acids. Comparison between the fragments obtained define the variation of sequences (Stojecki, 2012).

Taking into account the fact that in Romania this disease is still a major concern of public health, further studies are needed to better identify and characterize new methods that could help at the identification of Sarcocystissuihominis. Therefore the aim of our study was to determine the prevalence of Sarcocystissuihominis in pork obtained in the traditional households through PCR - RFLP method.

\section{MATERIALS AND METHODS Sample collection}

The material subjected to research was represented by seventy nine samples of diaphragmatic pillars isolated from pigs raised in the traditional system. The samples were collected between October 2014 and December 2014 from two local sanitary veterinary units from Alba and Cluj, located in the center of Transylvania. Meat samples had different sizes and were wrapped individually in plastic bags, separately for each animal. The samples were preserved by congelation, being transported for examination at the laboratory of «Inspection and food control of feed and animal origin products» in the Veterinary Medicine Faculty from Cluj - Napoca.

The collected sample were processed first by trichinelloscopic compression method, from eacharea mentioned abovethere were 28 tissue fragments displayed on the compressors' blade, treated with clarifying solution $(\mathrm{KOH} \mathrm{3 \%}$, $\mathrm{NaOH} 3 \%$ ) for a better high lightening of the microsarcocysts. The samples were examined with the screen trichinelloscope, in order to establish the presence or the absense of the Sarcocystis cysts. All the positive fragments for Sarcocystis spp. were examined through PCR-RFLP method.

\section{DNA extraction}

DNA extraction was performed by using a specific extraction kit Genomic ISOLATE II (Bioline), utilized for extraction from animal and human tissue and cell culture.

The first stage consisted of weighing out $25 \mathrm{mg}$ of muscle tissue from each sample and suspended in $180 \mu \mathrm{l}$ of Lysis Buffer GL and $25 \mu$ l Proteinase K solution in individual $1.5 \mathrm{ml}$ microcentrifuge tubes and then vortexed. The samples were incubated at $56^{\circ} \mathrm{C}$ in a termobloc for 3 hours and vortex every 
30 minutes, to complete lysis of the samples. After incubation, the samples were vortexed again. In each sample was added $200 \mu \mathrm{l}$ lysis solution (Lysis Buffer G3) and thenvortexed. After this step, the samples were incubated at a temperature of $70^{\circ} \mathrm{C}$ for 10 minutes and after the time were vortexed again. $210 \mu$ lethanol were added to each sample and then processed through a genomic DNA mini spin column containing a silica membrane to which the genomic DNA binds.

After washing the DNA, the „ISOLATE II Genomic DNA Spin Column - green" were introduced in $2 \mathrm{ml}$ tubes, then the sample was added and then centrifuged at 11,000 rpm for 60 seconds. The resulting supernatant was removed and the tubes were reutilized.

Contaminants and impurities such as salts, metabolites and cellular components are effectively removed by simple washing steps with two different buffers, adding first $500 \mu \mathrm{l}$ Wash Buffer GW1 and then centrifuged at 11,000 rpm for 60 seconds and second adding $600 \mu$ lWash Buffer GW2 while the same protocol for centrifugation was applied and the supernatant removed. After removing the liquid, the samples were again centrifuged for 1 minute at $11,000 \mathrm{rpm}$ in order to remove any ethanol left inside.

For each sample, the „ISOLATE II Genomic DNA Spin Column" was transferred in $1.5 \mathrm{ml}$ Eppendorf tubes and added $100 \mu \mathrm{l}$ preheated Elution Buffer $\mathrm{G},\left(70^{\circ} \mathrm{C}\right)$ was added directly onto the silica membrane. The sample was incubated at room temperature for 1 minute, then centrifuged for 1 minute at 11,000 rpm.

DNA samples resulting from the whole process were collected and stored in Eppendorff tubes.

\section{Detection and identification of Sarcocystis suihominis}

Detection and identification of Sarcocystissuihominis was performed using PCR-RFLP. The amplification of the target gene, $18 s r R N A$ gene was done using specific primers of sarF5'-GGA TAA CCT GGT AAT TCT ATG-3'and sarR 5'-GGC AAA TGC TTT CGC AGT AG-3' (Rosenthal, 2009). Based on databases, the amplicon size of $S$. Suihominis is $900 \mathrm{pb}$.

In order to apply the PCR protocol, samples were processed as following: in $200 \mu \mathrm{l}$ Eppendorff tubes were added $12.5 \mu \mathrm{l}$ MasterMix, $1 \mu \mathrm{l}$ Forward primer, $1 \mu \mathrm{l}$ Reverse primer, $4 \mu \mathrm{l}$ DNA and $6.5 \mu \mathrm{l}$ pure water, with a final volume of $25 \mu \mathrm{l}$.

Amplification of samples was carried out by using a Thermocycler (G-STORM). This stage involves subjecting samples to different temperatures in order to reconstitute the amplified DNA fragment.The amplification program was done with an initial denaturation of $95^{\circ} \mathrm{C}$ for $5 \mathrm{~min}$, followed by 45 cycles of denaturation at $95^{\circ} \mathrm{C}$ for $45 \mathrm{sec}$, annealing at $56^{\circ} \mathrm{C}$ for $45 \mathrm{sec}$ and extention at $72^{\circ} \mathrm{C}$ for $45 \mathrm{sec}$ with an elngation step of $5 \mathrm{~min}$ at $72{ }^{\circ} \mathrm{C}$ at the last cycle. The protocol ends by reaching a temperature of $4^{\circ} \mathrm{C}$.

RFLP analysis was performed using the AluI restriction enzyme, specific for Sarcocystis suihominis. The reaction was carried out by adding $2.5 \mu \mathrm{l}$ buffer solution, $5 \mu \mathrm{l}$ amplified DNA, $16 \mu \mathrm{l}$ pure water and $1.5 \mu \mathrm{l}$ AluI restriction enzyme, with a total reaction volume of $25 \mu$ l. Before adding the enzyme, the reaction components were vortexed, the enzyme being the last one added. After addition of enzyme, the sample is homogenized by mild pipetting.

After preparing the samples according to protocol, they were introduced in thermocycler at a temperature of $37^{\circ} \mathrm{C}$ for 5 minutes. To stop the reaction of DNA fragments restriction, the samples were maintained for 20 minutes at a temperature of $65^{\circ} \mathrm{C}$.

The electrophoretic profiles were carried out by the migration of the DNA samples in an agarose gel of $1.5 \%$ concentration. Reading and interpreting reaction was performed using the imaging system TransUV BioRad.

\section{Statistical interpretation}

For the interpretation of our results we used the OriginPro (Software version 8.5) and applied the ANOVA one-way and the least significant difference test. The Origin program is intended for plotting data and statistical analysis of biological experimentation with large or small data, integrating one or more variables.

\section{RESULTS AND DISCUSSION}

A total of 79 samples of pork meat were examined for the presence of microscopic sarcocysts.

After applying the compression method we have identified the presence of cysts of Sarcocystis spp. in the examined samples of diaphragm muscle 


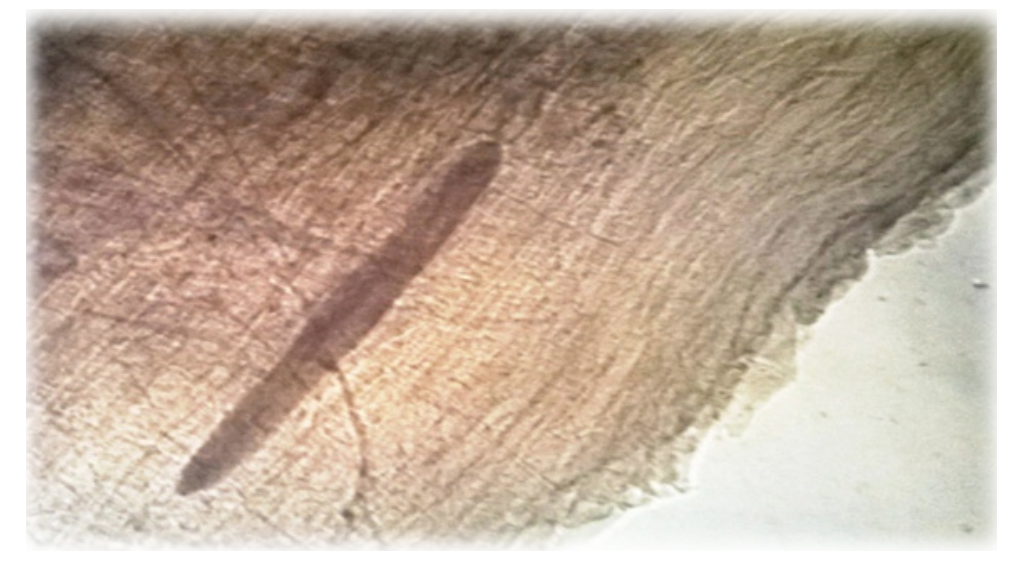

Fig. 1. Sarcocystis spp. cyst - the compression method on trichinoscope screen

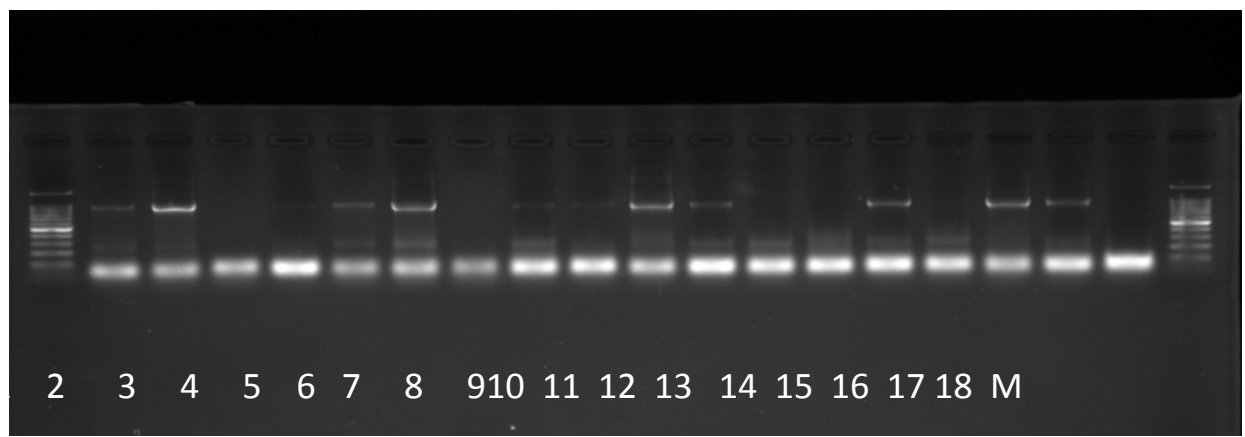

Fig. 2. The electrophoretic profile of $900 \mathrm{bp}$ fragment corresponding to Sarcocystis spp .; M - standard scale of 100 base pairs; Samples 2, 5, 6, 10,11, 14, 16, 17 - the evidence of the presence of specific DNA for Sarcocystis spp.

tissue in a procent of 72,15\%. The parasitic forms are shown in Figure 1.

After examining and identifying cysts in muscle tissue by compression exam we passed to the molecular identification of Sarcocystis spp. by PCR method. The simplex PCR method with specific primers revealed the fragmentcorresponding to common gene (18S) for all species of Sarcocystis, shown in Figure 2.

Using the AluI restriction enzyme we are allowed to distinguish between $S$. suihominis and the rest of the species of Sarcocystis. This enzyme has the ability to cut the fragment corresponding to this sub-species in two restriction sites and obtaining in the final three fragments at: 450,300 and 150 base pairs. This can be seen in Figure 3 where a significant part of the samples were restrictive and thus identified as positive for $S$. suihominis.

In this study, of the total samples examined (n $=79$ ), Sarcocystis spp. was molecular identified by PCR in $51.9 \%$ of cases.
In order to differentiateSarcocystis suihominis from the other Sarcocystis subspecies found in pigs we applied the RFLP method. Sarcocystis suihominis was found in a proportion of $51.2 \%$ in the samples molecular identified, the remaining $48.8 \%$ representing other subspecies of Sarcocystis with specificity and pathogenicity for pigs, but non-pathogenic to humans

After applying the entire protocol, the results have revealed the presence of $S$. suihominis in a high percentage of samples $(21.26 \%)$.

Based on the EC Regulation 854/2004 on official controls on meat intended for human consumption, the presence of Sarcocysts in the carcass of slaughtered animals is not covered by routine meat inspection. However, the high prevalence rate of infection, the presence of Sarcocysts species considered potentially zoonotic and the growing habit, in European countries, to eat raw or undercooked meat, brought some authors to consider the genus Sarcocystis as a 


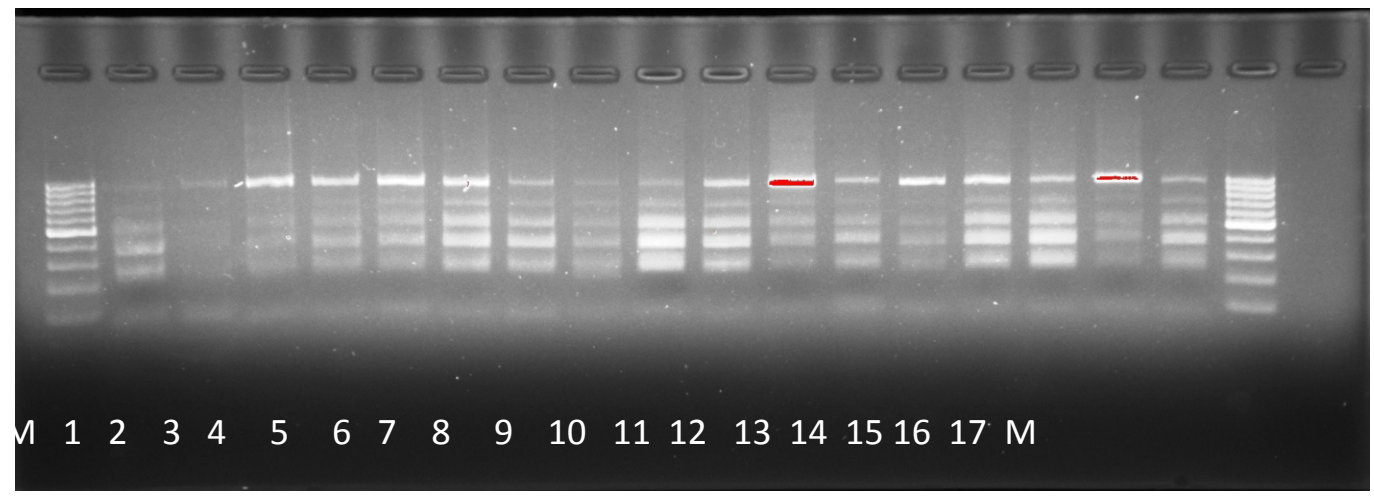

Fig. 3. The electrophoretic profile of restriction fragment of 400, 300, $150 \mathrm{bp}$ corresponding to Sarcocystis suihumonis; M - standard scale of 100 base pairs; Samples 1, 3, 4, 5, 6,7, 9, 10, 11, 12, 14, 15, 17 - The evidence of specific DNA restriction fragments for Sarcocystis suihumonis

possible zoonotic risk for consumers (Bucca et al., 2010; Chiesa et al., 2013).

Intestinal sarcocystosis in humans is fairly widespread in Europe, with a prevalence ranging from $1.6 \%$ to $10.4 \%$ (Dubey et al., 1989). These results are based on microscopic examination of fecal samples, a technique that cannot distinguish between S. suihominis and S. bovihominis, both of them being pathogenic to humans.

The estimated prevalence of Sarcocystis in pigs in central Europe is approximately 35\% for breeding animals and approximately $10 \%$ for fattening pigs (Daugschies, 2006). The results of a cross-sectional study on on the seroprevalence in breeding sows, carried in the German federal state of Hesse indicated a percentage of $25 \%$ of the tested animals positive (Damriyasa et al., 2004). Species specification was not possible in this study because of cross-reactivity of the antigen used in the test, though it was suspected that S. suihominis is probably more common. Older studies, carried out between 1963 and 1974, reviewed in Heydorn (1977) and 1978 to 1994 (cited in Damriyasa et al., 2004) report infections of $2.8 \%$ - $60 \%$ in Germany, $1.1 \%$ - 95\% in Poland, 7.4\% - 32\% in Austria, 25\% in Hungary, $10.5 \%$ in Bulgaria, $1.8 \%-3.5 \%$ in Denmark, $18 \%$ in Iowa (USA), and 16\% in Japan. Where results were differentiated, the reported prevalence in older animals (sows and boars) was higher compared to fattening pigs. However, the differences and wide percentage ranges were not only considered the result of using various detection methods but also significantly influenced by differences in husbandry and hygiene factors (Taylor et al., 2010).
A study of literature before 2000 revealed a single report using PCR-RFLP technique to investigate the diversity of the genus Sarcocystis; this study involved isolating $S$. gigantean from two slaughterhouses (Jeffries et al., 1996), without there being a report subsequently published about the interspecific variation of the parasites of this kind. The prevalence of this parasite in meat has been declared in many countries (Ginawi and Shommein, 1977), but unfortunately a proper investigation in Romania has not yet been published. Clinical signs of human intestinal sarcocistosis are particularly digestive system disorders such as nausea, vomiting, diarrhea (Dubey et al., 1989), which appeared especially in immunocompromised patients (Velásquez et al., 2008). According to data released by Saleque et al. (1990), intestinal sarcocystosis can be prevented by thoroughly cooking or freezing meat to disablethe action of bradyzoites from the sarcocysts. Sarcocysts in pig muscles became noninfectious for puppies after cooking meat at 60 , 70 , and $100^{\circ} \mathrm{C}$ for 20,15 , and $5 \mathrm{~min}$, respectively. Freezing at $-4^{\circ} \mathrm{C}$ and $-20^{\circ} \mathrm{C}$, for 48 and $24 \mathrm{~h}$, respectively, also rendered bradyzoites in pork noninfectious (Fayer et al., 2004).

Visual inspection at the slaughterhouse will identify macroscopic lesions, not necessarily caused by the zoonotic species, but does not allow species differentiation. Species differentiation can be carried out using various methods (Taylor et al., 2010).

In the last decade, nearly all molecular approaches to the detection and diagnosis of species of Sarcocystis were based on the amplification and characterization of ribosomal 
DNA. Such methods have differentiated among parasites localized in the tissues of various livestock (and wildlife) hosts, including those of swine and cattle. For S.hominis and S.suihominis, such molecular methods have been applied to the encysted forms found in beef and swine muscle.

Thus, Sarcocystis hominis and S.suihominis represent just two out of hundreds of species mentioned in the Sarcocystidae, which also includes parasites variously assigned to the genera Toxoplasma, Besnoitia, Frenkelia, Hammondia, Neospora, and Sarcocystis. For every study of $S$. horninis or S. suihominis, more than 100 have been published for Toxoplasmagondii owing to its especially clinical significance as a major threat to human reproductive health and a serious opportunistic pathogen of AIDS. This justifiably disproportionate research effort has resulted in a suite of experimental methods and assays, including tools of modern molecular genetics, immunological reagents, in vitro culture protocols, in vivo phenotyping, genome sequences, population genetics, expression analysis, and more.

Molecular methods can and should be employed to aid diagnosis of human infection with S.hominis and S.suihominis. Such methods, moreover, offer great potential in exploring the evolutionary origins and exposure routes of other human parasites whose identity and provenance remains enigmatic (Rosenthal, 2009).

Molecular techniques should become a routine, because they can be applied directly to identify certain species of Sarcocystis.

\section{CONCLUSIONS}

The results of this study demonstrate how the PCR-RFLP method, developed in this study, represents an effective and rapid method for the identification of Sarcocystis suihominis in pork meat. It is recommended that the consumption of pork meat and meat products to occur after an appropriate heat treatment to avoid the risk of contamination by eating pork produced in the traditional system, because from the survey conducted the Sarcocystis suihominis was detected in the analyzed samples in a percentage of $26.58 \%$.

\section{REFERENCES}

1. Acha PN, Szyfres B (2003). Zoonoses and communicable diseases common to man and animals, 3rd ed., vol.
III: Parasitoses, Pan American Health Organization, Washington D.C.

2. Bucca M, Brianti E, Giuffrida A, Ziino G, Cicciari S, Panebianco A (2010). Prevalence and distribution of Sarcocystis spp. cysts in several muscles of cattle slaughtered in Sicily, Southern Italy. Food Control 22:1058.

3. Cătoi C (2003). Diagnostic necropsic veterinar. Editura AcademicPres, Cluj-Napoca, p. 255.

4. Chiesa F, Muratore E, Dalmasso A, Civera T (2013). A new molecular approachto assess the occurrenceof Sarcocystis spp. in cattleand products thereof:preliminary data. Italian Journal of Food Safety; volume 2:e41.

5. Constantin N (2014). Tratat de medicină veterinară, Volumul VI. Editura Risoprint, Cluj-Napoca, p. 321-335.

6. Dahlgren SS, Gjerde B (2008). Sarcocystis in moose (Alces alces): molecular identification and phylogeny of six Sarcocystis species in moose, and a morphological descriptionof three new species. Parasitol Res 103: 93110.

7. Damriyasa IM, Bauer C, Edelhofer R, Failing $K$, Lind $P$, Petersen E, Schares G, Tenter AM, Volmer R, Zahner H (2004). Cross-sectional survey in pig breeding farms in Hesse, Germany: seroprevalence and risk factors of infections with Toxoplasma gondii, Sarcocystisspp. and Neosporacaninumin sows. Veterinary Parasitology 126:271-286

8. Daugschies A (2006). Protozoeninfektionen des Schweines. Editura Schnieder T., Veterinär medizinische Parasitologie. PareyVerlag, Berlin, p. 359-368

9. Dubey JP, Odening K (2001). Toxoplasmosis and Related Infections. In: Samuel, W.M., Pybus, MJ, Kocan AA (Eds.), Parasitic Diseases of Wild Mammals. Iowa State University Press, Ames, pp. 478-511.

10. Dubey JP, Speer CA, Fayer R (1989). Sarcocystis of animals and man. CRC Press, USA, p.215

11. Fayer R (2004). Sarcocystisspp. in Human Infections. Clincal Microbiology Reviews 17(4):894-902.

12. Ginawi MA, Shommein AM (1977). Prevalence of sarcosporidiosis in sheep, goats and camels in Sudan. Sudan J Vet Sci\&AnimHusb 18:92-97.

13. Güçlü F, Aldem R OS, Güler L (2004). Differential identification of cattle Sarcocystis spp. by random amplified Polymorphic DNA - Polymerase chain reaction (RAPD-PCR). Revue Méd. Vét.155:440-4.

14. Heydorn AO (1977). Sarkosporidien infiziertes Fleisch als mögliche Krankheitsursache für den Menschen. Archiv für lebensmittel hygiene, 28:27-31.

15. Jeffries AC, Amaro N, Tenter AM, Johnson AM (1996). Genetic diversity in Sarcocystis gigantea assessed by RFLP analysis of theITS1 region. Applied Parasitology 37:275283.

16. Juyal PD, Bhatia BB (1989). Sarcocystosis: An emerging zoonosis, Indian Veterinary Medical Journal, 13: 66-69.

17. Prakas P, Butkausas D (2012). Protozoan parasites from genus Sarcocystis and their investigation in Lithuania. Ekologija58(1):45-58. 
18. Rosenthal BM (2009). Molecular Detection of Food borne Pathogens. CRC Pres, United States Department of Agriculture, p. 735-736

19. Rosenthal BM, Dunams DB, Pritt B (2008) Restricted genetic diversity in the ubiquitous cattle parasite, Sarcocystiscruzi. Infect Genet Evol 8:588-592.

20. Saleque A, Bhatia BB (1991). Prevalence of Sarcocystis in domestic pigs in India. Vet Parasitol 40: 151-153.

21. Saleque A, Juyal PD, Bathia BB (1990). Effect of temperature on the infectivity of Sarcocystismieschenariana cysts in pork. Veterinary Prasitology 36: 343.

22. Stojecki K (2012). Molecular diagnostics of Sarcocystisspp. infections. Polish Journal of Veterinary Sciences 15(3):589-596.

23. Taylor MA, Boes J, Boireau P, Boué F, Claes M, Cook A JC, Dorny P, Enemark H, Joke van der Giessen, Hunt KR, Howell M, Kirjušina M, Nöckler K, Pozio E, Rossi P, Snow L, Theodoropoulos G, Vallée I, Vieira-Pinto MM, Zimmer
IA (2010). Development of harmonised schemes for the monitoring and reporting of Sarcocystis in animals and foodstuffs in the European Union. EFSA Supporting Publication 2010; 7(1):EN-33, 28 pp. doi:10.2903/ sp.efsa.2010.EN-33

24. Tenter AM (1995). Current research on Sarcocystis species of domestic animals. Int J Parasitol 25:1311-30.

25. Velásquez JN, Di Risio C, Etchart CB, Chertcoff AV, Mendez N, Cabrera MG, Labbe JH, Carnevale S (2008). Systemic sarcocystosis in a patient with acquired immune deficiency syndrome. Human Pathology 39(8):1263-1267.

26. World Health Organization (1981) Intestinal protozoan and helminthic infections, vol. 666. World Health Organization, Geneva.

27. Xiang Z, Chen X, Yang L, He Y, Jiang R, Rosenthal BM, Luan $P$, Attwood SW, Zuo Y, Zhang YP, Yang Z (2009) Non-invasive methods for identifying oocysts of Sarcocystis spp. from definitive hosts. Parasitol Int 58: 293-296. 